\title{
Isothermal titration calorimetry (ITC) study of natural cyclodextrins inclusion complexes with tropane alkaloids
}

\author{
Małgorzata Wszelaka-Rylik ${ }^{1} \cdot$ Paweł Gierycz $^{2,3}$
}

Received: 3 December 2014 / Accepted: 18 March 2015/Published online: 4 April 2015

(c) The Author(s) 2015. This article is published with open access at Springerlink.com

\begin{abstract}
Isothermal titration calorimetry (ITC) has been used to characterize inclusion complex formation of natural cyclodextrins ( $\alpha$ - and $\beta$-cyclodextrin) with three tropane alkaloids (scopolamine, homatropine hydrobromide and atropine sulfate) in aqueous solutions. ITC measurements were taken at $298.15 \mathrm{~K}$ on a MicroCal OMEGA ultrasensitive titration calorimeter (MicroCal Inc.). The experimental data were analyzed on the basis of the model of a single set of identical sites (ITC Tutorial Guide). $\beta$-CD forms inclusion complexes of stoichiometry $1: 2$ with homatropine hydrobromide and 1:1 with scopolamine and atropine sulfate. The smaller molecule of $\alpha$-CD forms very weak inclusion complexes with the tropane alkaloids. So, only one complex of the same stoichiometry 1:2 with homatropine hydrobromide has been detected by the ITC experiment. Based on the experimental values of equilibrium constant $(K)$ and enthalpy of complex formation $(\Delta H)$, the Gibbs energy of complex formation $(\Delta G)$ and the entropy of complex formation $(\Delta S)$ have been calculated, for all the investigated systems. Obtained results showed that complex formation of both $\alpha$ - and $\beta-C D$ with all the investigated tropane alkaloids is entropy driven. This indicated that the difference in the cavity dimensions is not
\end{abstract}

Małgorzata Wszelaka-Rylik

m.wszelaka-rylik@uksw.edu.pl

1 Faculty of Biology and Environmental Sciences, Cardinal Stefan Wyszynski University in Warsaw, Wóycickiego 1/3, 01-938 Warsaw, Poland

2 Institute of Physical Chemistry, Polish Academy of Science, Kasprzaka 44/52, 01-224 Warsaw, Poland

3 Faculty of Chemical and Process Engineering, Warsaw University of Technology, Warynskiego 1 , 00-645 Warsaw, Poland reflected in different driving forces of complex formation and binding modes which resulted in the same stoichiometry of the obtained inclusion complexes.

Keywords Isothermal titration calorimetry (ITC) . $\alpha$-Cyclodextrin · $\beta$-Cyclodextrin - Scopolamine . Homatropine hydrobromide - Atropine sulfate . Inclusion complexes

\section{Introduction}

Cyclodextrins (CDs), the cyclic oligosaccharides, have recently been recognized as useful pharmaceutical excipients. The most common are natural, cyclic oligosaccharides containing six ( $\alpha$-cyclodextrin: $\alpha-C D)$, seven $(\beta-$ cyclodextrin: $\beta-\mathrm{CD})$ or eight $(\gamma$-cyclodextrin: $\gamma-\mathrm{CD}) \alpha-1,4-$ linked glucopyranose units, with a hydrophilic hydroxyl group on their outer surface and a hydrophobic cavity in the center.

The CDs, dependent on the arrangement of their functional groups leading to the formation of a relatively hydrophilic outside and hydrophobic internal cavity, possess the ability to form inclusion complexes with many drugs by taking up a whole drug molecule, or some part of it, into the cavity. The complexation depends largely on the dimensions of the CDs and the particular sterical arrangement of its functional groups. Driving forces of the complexation process are both Van der Waals interactions and hydrophobic effect. In addition, hydrogen bonds at the rim of the cavity may also play a certain role $[1,2]$.

The complexation depends on the phase in which it takes place. In the solid state, guest molecules can be enclosed within the cavity or may be aggregated to the outside of the $\mathrm{CD}$ molecule, while in the liquid state (a 
solution), we deal with an equilibrium between complexed and non-complexed guest molecules.

One of the most interesting properties of CDs is the formation of inclusion complexes with a wide variety of guest molecules. The CDs inclusion complexes affect many physicochemical properties of their quests, such as aqueous solubility and rate of dissolution. The successful enhancing of the solubility of poorly water-soluble drugs belongs to the most important application of such complexes [3-6].

A guest molecule changes its physicochemical properties when it is incorporated within the CD cavity. The techniques used for characterization of inclusion complexes formed between the guest and CD molecules can be divided into two groups depending on the complex state (solid or liquid-solution) [7]. One of the very useful techniques for characterization of inclusion complexation in solution is microcalorimetry. It detects changes in thermodynamic properties (enthalpy and entropy) due to inclusion complexation. These changes are associated with the change in the behavior of water structure within the cavity, removal of water from the cavity, restructuring of water around the guest molecule and release of water into the bulk.

The aim of this work, which is a part of a big project concerning investigation of complexes formed by drugs and CDs [8], is the isothermal titration calorimetry (ITC) study of inclusion complexes formed by natural CDs $(\alpha-$ $\mathrm{CD}$ and $\beta-\mathrm{CD})$ with three tropane alkaloids: scopolamine, homatropine hydrobromide and atropine sulfate.

The ITC method has been widely used for characterization of drug inclusion complexes with natural CDs [9-16], but none of the experiments have dealt with the investigated drugs.

Inclusion complexes of $\beta$-CD with scopolamine have been studied ( $\left.{ }^{1} \mathrm{H}-\mathrm{NMR}\right)$ by Blaschke et al. [17], but in the literature there is the lack of direct information concerning thermodynamic properties of the studied complexes.

\section{Experimental}

\section{Materials and solutions}

All CDs were provided by Wacker Chemie AG (Germany) and were of pharmaceutical grade $(\alpha-\mathrm{CD}$ : Cavamax W6 Pharma; $\beta-C D$ : Cavamax W7 Pharma). Before experiments, they were dried in vacuum drier SPU-200 (ZUT COLECTOR, Poland) for $24 \mathrm{~h}$.

All the drugs, i.e., scopolamine, homatropine hydrobromide and atropine sulfate, were supplied by Sigma (Steinheim, Germany). They were of analytical grade and for the experiments were used as received.
For the calorimetric experiments, both CDs and drugs were dissolved in water and degassed. The initial concentrations of the all CDs were equal to $1.5 \mathrm{mM}$ and the alkaloids to $30 \mathrm{mM}$.

\section{Methods}

ITC measurements were taken at $298.15 \mathrm{~K}$ on a MicroCal OMEGA ultrasensitive titration calorimeter (MicroCal Inc.). The parameters of the titration (the number-31, volume $-8 \mu \mathrm{L}$ and length of time of injections) were input into the software program controlling data. The aqueous solutions in the cell were stirred by the syringe at $400 \mathrm{rpm}$. The volume of a titrant was injected over $30 \mathrm{~s}$ with an interval of $240 \mathrm{~s}$ between injections from a $250-\mu \mathrm{L}$ injection syringe into the sample cells (containing CDs) in a series of controlled pulses. The sample cell volume was $1.3611 \mathrm{~mL}$. The integrated heat effects of each injection were corrected by subtraction of the corresponding integrated heat effects of dilution of the drug and the cyclodextrin, respectively, i.e., heat effect of injection of drug solution to the water and heat effect of water injection to the cyclodextrin solution.

The experimental data obtained from the calorimetric titration were analyzed on the basis of the model of a single set of identical sites (ITC Tutorial Guide).

\section{Results}

Isothermal titration calorimetry experiments have been performed at $298.15 \mathrm{~K}$ for the six, mentioned above, following systems:

1. $\alpha$-cyclodextrin + scopolamine,

2. $\alpha$-cyclodextrin + homatropine hydrobromide,

3. $\alpha$-cyclodextrin + atropine sulfate,

4. $\beta$-cyclodextrin + scopolamine,

5. $\beta$-cyclodextrin + homatropine hydrobromide,

6. $\beta$-cyclodextrin + atropine sulfate.

To ensure the accuracy of the results, each ITC experiment was repeated five times with the difference between the obtained equilibrium constants in each measurement $<3 \%$.

The obtained results of the ITC experiments are given in Table 1 and Figs. 1 and 2. Table 1 shows the equilibrium constants $(K)$ and enthalpies of complex formation $(\Delta H)$ calculated by the model of a single set of identical sites (ITC Tutorial Guide) for all the investigated inclusion complexes, and Figs. 1 and 2 present the curve fitting of the enthalpy of complex formation for selected systems. 
Table 1 Equilibrium constants $(K)$ and enthalpy of complex formation $(\Delta H)$ for all the investigated inclusion complexes

\begin{tabular}{|c|c|c|c|c|}
\hline \multirow[t]{2}{*}{ Compound } & \multicolumn{2}{|l|}{$\alpha$-Cyclodextrin } & \multicolumn{2}{|l|}{$\beta$-Cyclodextrin } \\
\hline & $K / \mathrm{mole}^{-1}$ & $\Delta H / \mathrm{kcal} \mathrm{mole}^{-1}$ & $K / \mathrm{mole}^{-1}$ & $\Delta H / \mathrm{kcal} \mathrm{mole}^{-1}$ \\
\hline Scopolamine & - & - & $1.5210^{3} \pm 110$ & $-1.04 \pm 0.12$ \\
\hline Homatropine hydrobromide & $0.6210^{3} \pm 58$ & $-0.08 \pm 0.01$ & $1.2110^{3} \pm 92$ & $-0.39 \pm 0.04$ \\
\hline Atropine sulfate & - & - & $6.0610^{3} \pm 190$ & $-1.17 \pm 0.01$ \\
\hline
\end{tabular}

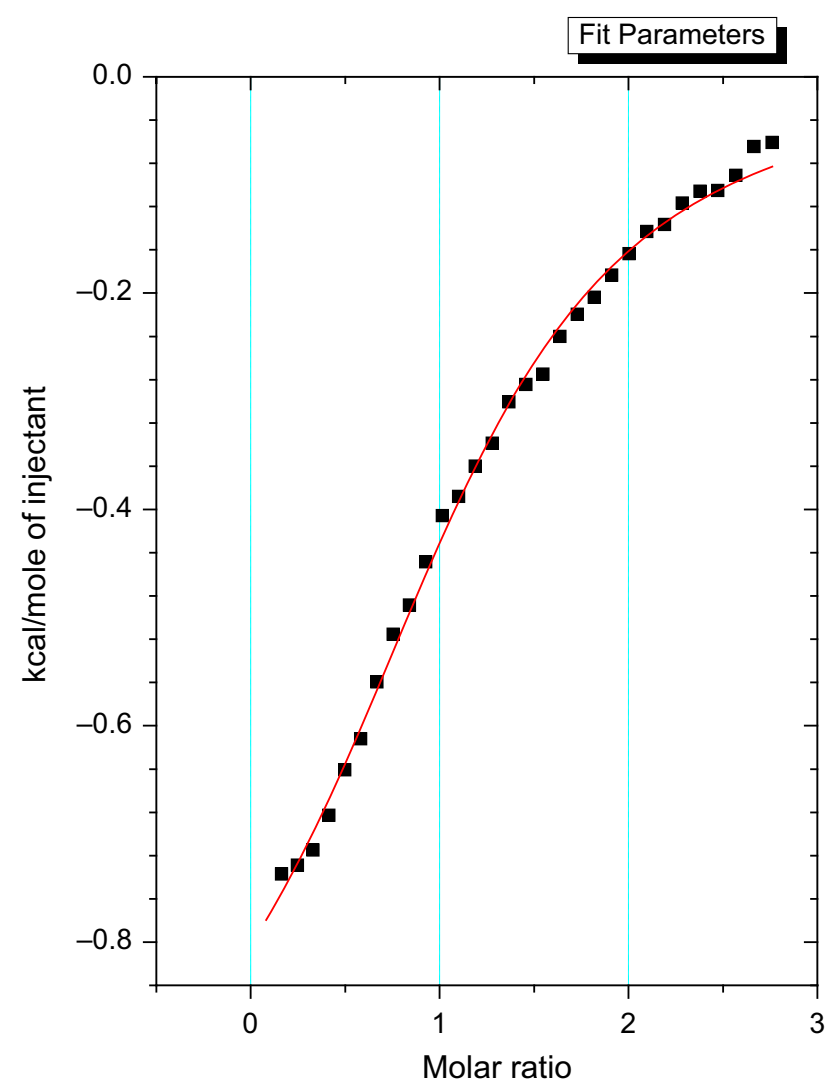

Fig. 1 ITC result for a titration of a $\beta$-cyclodextrin water solution against scopolamine water solution. Axis $X$ : "molar ratio" means " $\beta$ cyclodextrin:scopolamine molar ratio"

Looking at the results of an ITC experiment for $\beta-C D$ scopolamine (Fig. 1; Table 1), it is seen that although concentration of solution applied was relatively low, there was still a significant release of heat detectable. This effect points to a high equilibrium constant (Table 1). Curve fitting of the enthalpy of complex formation ( $\Delta H$-Fig. 1) plot revealed, in this case, best accordance with a stoichiometry of 1:1 ( $\beta$ $\mathrm{CD}$ :scopolamine). The only data, concerning a stoichiometry of $\beta-C D$ with scopolamine, could be found in the Blaschke et al. paper [17]. The authors estimated the complex stoichiometry from ${ }^{1} \mathrm{H}-\mathrm{NMR}$ data, based on the continuous variation method [18], and found out that the Job plot (both aromatic protons and $\mathrm{H}$ proton) for $\beta-\mathrm{CD}+$ scopolamine system had the symmetrical shape which results in stoichiometry of 1:1 ( $\beta$-CD:scopolamine). So, the result

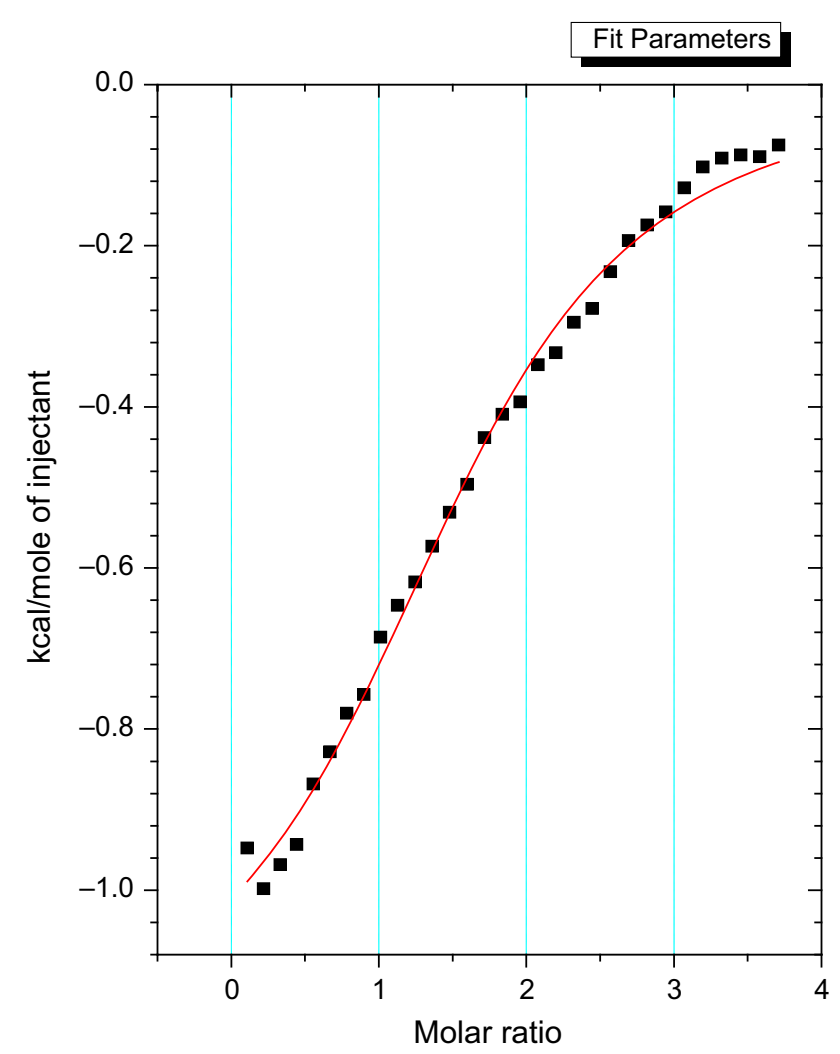

Fig. 2 ITC result for a titration of a $\beta$-cyclodextrin water solution against atropine sulfate water solution. Axis $X$ : "molar ratio" means " $\beta$-cyclodextrin:atropine sulfate molar ratio"

confirms and fully agrees with our investigations concerning the obtained stoichiometry of $1: 1$ in the case of complex formation of $\beta-\mathrm{CD}$ with scopolamine.

However, it is a big discrepancy between calculated values of the equilibrium constant of the investigated $\beta$ $\mathrm{CD}+$ scopolamine complex and the literature data [17]. The values of $K$ constant (Table 2) calculated, based on the very well fit (Fig. 1) of the enthalpy of complex formation, are about 50 times higher than the equilibrium constant calculated by the use of the Benesi-Hildebrand method [19], from the ${ }^{1} \mathrm{H}-\mathrm{NMR}$ data [17]. It is necessary to notice that the value of $K$ calculated from the ${ }^{1} \mathrm{H}-\mathrm{NMR}$ data [17] was obtained by the procedure [19] which is very imprecise for protons which do not experience large shifts upon complex formation or for signals which are partially obscured by other peaks [17]. 
Table 2 Gibbs energy of complex formation $(\Delta G)$ and the entropy of complex formation $(\Delta S)$ for the all investigated systems

\begin{tabular}{|c|c|c|c|c|}
\hline \multirow[t]{2}{*}{ Compound } & \multicolumn{2}{|l|}{$\alpha$-Cyclodextrin } & \multicolumn{2}{|l|}{$\beta$-Cyclodextrin } \\
\hline & $\Delta G / \mathrm{kcal} \mathrm{mole}^{-1}$ & $T \Delta S / \mathrm{kcal} \mathrm{mole}^{-1}$ & $\Delta G / \mathrm{kcal} \mathrm{mole}^{-1}$ & $T \Delta S / \mathrm{kcal} \mathrm{mole}^{-1}$ \\
\hline Scopolamine & - & - & $-4.34 \pm 0.05$ & 3.30 \\
\hline Homatropine hydrobromide & $-3.81 \pm 0.06$ & 3.73 & $-4.21 \pm 0.05$ & 3.81 \\
\hline Atropine sulfate & - & - & $-5.16 \pm 0.04$ & 3.99 \\
\hline
\end{tabular}

The obtained results of ITC experiments for the inclusion complexes formed by $\beta-C D$ with two other tropane alkaloids (homatropine hydrobromide and atropine sulfate) as well as $\alpha$-CD with homatropine hydrobromide are given in Table 1 and Fig. 2 (curve fitting of the enthalpy of complex formation for $\beta$-CD and atropine sulfate-for the three tropane alkaloids, the enthalpy curves were very similar, so the only one example is given).

Curve fitting of the enthalpy of complex formation ( $\Delta H$-Fig. 2) plot revealed, in all the cases, best accordance with a stoichiometry of $1: 2(\beta-\mathrm{CD}$ or $\alpha$-CD:tropane alkaloid). In the literature, there is a lack of reliable data concerning the equilibrium constants, the enthalpies of complex formation and the stoichiometry. However, very precise and accurate fit of the experimental enthalpy of complex formation of those systems allows for assumption that one can rely on the obtained experimental ITC data.

It is necessary to point out that in the case of atropine sulfate we deal with two atropine particles in one atropine sulfate particle. So, the stoichiometry of 1:2 ( $\beta$-CD:tropane alkaloid) results in the case of $\beta-\mathrm{CD}$ and atropine sulfate in the stoichiometry of 1:1 ( $\beta$-CD:atropine).

Looking at the results of an ITC experiment for $\alpha-C D$ and all the investigated drugs (Table 1), it is seen that $\alpha$ $\mathrm{CD}$ forms inclusion complex only with homatropine hydrobromide. In the case of other two drugs, i.e., scopolamine and atropine sulfate, none of the characteristic effects for formation of inclusion complexes was observed. The detected enthalpy of complex formation of $\alpha-\mathrm{CD} / \mathrm{s}-$ copolamine (and atropine sulfate) was constant and, on the level of the method accuracy, equal to the sum of dilution and mixing effects of $\alpha-\mathrm{CD} /$ water and water/scopolamine (and atropine sulfate).

The absolute value of inclusion complex formation enthalpy of $\alpha$-CD with homatropine hydrobromide (Table 1) was the lowest from all the complexes investigated and was about five times lower than the lowest from among $\beta-C D$ complexes with the tropane alkaloids $(\beta-C D+$ homatropine hydrobromide-Table 1). It results in a much lower equilibrium constant for this system (more than two times lower than $K$ for $\beta$-CD complex with homatropine hydrobromide-Table 1).

It is necessary to take into account that although the size of homatropine hydrobromide and scopolamine is similar,
$\alpha$-CD forms weak complex only with homatropine hydrobromide (Table 1). Moreover, in this case, despite the fact that for small-sized molecules the difference in cavity diameter of $\alpha$ - and $\beta$-CD usually plays major role in complexation [20], both $\alpha$ - and $\beta$-CD form the complexes with the same stoichiometry of $1: 2$ ( $\beta-\mathrm{CD}$ or $\alpha$-CD:tropane alkaloid). It is necessary also to notice that the weakest interaction (the lowest equilibrium constants) has been measured for the $\alpha$ - and $\beta$-CD complex formation with homatropine hydrobromide (Table 1).

The heat detectable for $\beta$-CD complex formation with homatropine hydrobromide significantly differs from the heat obtained for the $\beta-\mathrm{CD}+$ scopolamine complex (Table 1). The absolute value of the heat is about four times lower than in the case of scopolamine (Table 1) which results in a lower equilibrium constant (Table 1). In the case of $\beta-C D+$ atropine sulfate complex formation, we deal with the opposite situation. The absolute value of the heat detected, in this case, is of about $10 \%$ higher than in the case of scopolamine (Table 2), but the equilibrium constant is much (almost four times) higher than the equilibrium constant of $\beta-\mathrm{CD}+$ scopolamine complex (Table 1).

Based on the values of equilibrium constant $(K)$ and enthalpy of complex formation $(\Delta H)$, the Gibbs energy of complex formation $(\Delta G)$ and the entropy of complex formation $(\Delta S)$ have been calculated, for all the investigated systems, from the following equations:

$$
\begin{aligned}
& \ln K=-\frac{\Delta G}{R T} \\
& \Delta G=\Delta H-T \Delta S
\end{aligned}
$$

where $T$ is the temperature $[\mathrm{K}]$ and $R$ is the gas constant $\left(\mathrm{J} \mathrm{mol}^{-1} \mathrm{~K}^{-1}\right)$.

The calculated values of the Gibbs energy and the entropy of complex formation for the all investigated systems are given in Table 2.

Results displayed in Tables 1 and 2 clearly show that complex formation of both $\beta$-CD (bigger molecule with wider cavity compared with $\alpha-\mathrm{CD}$ ) with all the investigated tropane alkaloids (scopolamine, homatropine hydrobromide and atropine sulfate) and $\alpha-C D$ with homatropine hydrobromide is entropy driven. This indicates that the difference in the cavity dimensions is not reflected in 
different driving forces of complex formation and binding modes which results in the same stoichiometry of the obtained inclusion complexes.

Looking at the obtained results (Table 1), it is seen that the complexation of both $\alpha-C D$ and $\beta-C D$ with homatropine hydrobromide is much less exothermic than the complexation of $\beta-C D$ with the other (scopolamine and atropine sulfate) tropane alkaloids. It means that in this case the weaker (compared with the other investigated alkaloids) van der Waals interaction between CDs and homatropine hydrobromide takes place. If we have a weak interaction (low value of $K$ ), the particle does not enter the cavity (or enters it only slightly). That is why, in this case, the $\mathrm{CD}$ ring can interact not only with one particle but also with two particles and 1:2 complex can be formed.

$\beta$-CD complex formation with the other investigated drugs is more exothermic compared with the complexation with homatropine hydrobromide (Table 1). It is probably caused by the fact that exothermic effects from van der Waals interaction, H-bonding and the expulsion of cavitybound water in these cases are much less overlapped by endothermic effects connecting with dehydration and hydrophobic interaction.

It is necessary to point out the positive change in entropy of the obtained complexes. The breakage of hydration shells and release of water molecules to the bulk produce the positive contribution to $\Delta H$ and $\Delta S$ of the complexes.

\section{Conclusions}

The analysis of investigation results of the inclusion complexes shows that there is a big difference between the equilibrium constants and the enthalpy of complex formation of $\alpha$ - and $\beta-C D$ with homatropine hydrobromide. The absolute value of enthalpy of $\alpha-C D+$ homatropine hydrobromide inclusion complex formation is about five times lower than the absolute value of enthalpy of $\beta-C D+$ homatropine hydrobromide complex formation (Table 2) which results in big (about 2.5 times) difference in values of the equilibrium constant of those complexes (Table 1).

It is necessary to point out that the thermodynamic properties of $\alpha$ - and $\beta$-CD with homatropine hydrobromide differ significantly asunder and from the other investigated complexes. Both the enthalpy and the equilibrium constants of the $\beta-C D$ inclusion complexes with the other tropane alkaloids (scopolamine and atropine sulfate) are much higher (Table 1) than the values obtained for the complex of $\beta-C D$ with homatropine hydrobromide. In the case of the $\beta-C D$ complex with atropine sulfate, the enthalpy of complex formation is about $10 \%$ higher than in the case of the $\beta$-CD complex with scopolamine and about eight times higher than the value for the $\beta-\mathrm{CD}$ complex with homatropine hydrobromide (Table 1).

It is interesting to notice the positive and similar value change in entropy of the all obtained complexes (Table 2) causing that the complex formation of both $\beta$-CD (bigger molecule with wider cavity compared with $\alpha$-CD) with all the investigated tropane alkaloids (scopolamine, homatropine hydrobromide and atropine sulfate) and $\alpha-C D$ with homatropine hydrobromide is mainly entropy driven.

The different thermodynamic parameters $(K, \Delta H$, $\Delta G$ and $\Delta S$ ) of the investigated systems are not connected with the stoichiometry of the formed inclusion complexes. Both the bigger molecule (higher cavity diameter) of $\beta-C D$ and the smaller molecule of $\alpha-C D$ form inclusion complexes of stoichiometry 1:2 with homatropine hydrobromide. However, it is important to notice that the $\beta$-CD forms the strong (high value of the equilibrium constantTable 1) inclusion complexes with all the investigated tropane alkaloids, while the $\alpha$-CD forms the weak (low value of the equilibrium constant-Table 1) inclusion complex only with homatropine hydrobromide.

Experimental results obtained for the inclusion complexes formed by natural CDs with drugs confirmed applicability of the ITC method for both proper analysis of that type of complexes and direct determination of their thermodynamic properties.

Acknowledgements The work was partially supported by the European Union within European Regional Development Fund, through Grant Innovative Economy (POIG.01.01.02-14-102/09).

Open Access This article is distributed under the terms of the Creative Commons Attribution License which permits any use, distribution, and reproduction in any medium, provided the original author(s) and the source are credited.

\section{References}

1. Rekharsky MV, Inoue Y. Complexation thermodynamics of cyclodextrins. Chem Rev. 1998;98:1875-917.

2. Del Valle EMM. Cyclodextrins and their uses: a review. Process Biochem. 2004;39:1033-46.

3. Loftssan T, Brewster ME. Pharmaceutical application of cyclodextrins 1. Solubilization and stabilization. J Pharm Sci. 1996;85:1017-25.

4. Rajewski RA, Stella VJ. Pharmaceutical application of cyclodextrins 2. In-vivo. Drug Delivery. J Pharm Sci. 1996;85:1142-69.

5. Kozbiał M, Gierycz P. Comparison of aqueous and 1-octanol solubility as well as liquid-liquid distribution of acyclovir derivatives and their complexes with hydroxypropyl-b-cyclodextrin. J Solut Chem. 2013;42:866-81.

6. Kozbiał M, Gierycz P. Partitioning and complexation study of bioactive tricyclic acyclovir derivative with cyclodextrins. J Chem Thermodyn. 2014;72:23-30.

7. Singh R, Bharti N, Madan J, Hiremath SN. Characterization of cyclodextrin inclusion complexes-a review. J Pharm Sci Technol. 2010;2:171-83. 
8. Wszelaka-Rylik M, Gierycz P. Isothermal titration calorimetry (ITC) study of natural cyclodextrins inclusion complexes with drugs. J Therm Anal Calorim. 2013;111(3):2029-35.

9. Muller BK, Ritter H. Scrutinizing ITC-study on the formation of inclusion complexes of nonionic surfactant Triton X-100 and cyclodextrins. J Incl Phenom Macrocycl Chem. 2011; doi:10. 1007/s10847-011-9955-0.

10. Stojanov M, Wimmer R, Larsen KL. Study of the inclusion complexes formed between cetirizine and $\alpha-, \beta$-, and $\gamma$-cyclodextrin and evaluation on their taste-masking properties. J Phar Sci. 2011;100:3177-85.

11. Bouchemal K. New challenges for pharmaceutical formulations and drug delivery systems characterization using isothermal titration calorimetry. Drug Discov Today. 2008;13:960-72.

12. Castronuovo G, Niccoli M. Thermodynamics of inclusion complexes of natural and modified cyclodextrins with propranolol in aqueous solution at $298 \mathrm{~K}$. Bioorg Med Chem. 2006;14:3883-7.

13. Denadai AM, Teixeira KI, Santoro MM, Pimenta AM, Cortes ME, Sinisterra RD. Supramolecular self-assembly of beta-cyclodextrin: an effective carrier of the antimicrobial agent chlorhexidine. Carbohydr Res. 2007;342:2286-96.

14. Illapakurthy AC, Wyandt CM, Stodghill SP. Isothermal titration calorimetry method for determination of cyclodextrin complexation thermodynamics between artemisinin and naproxen under varying environmental conditions. Eur J Pharm Biopharm. 2005;59:325-32.
15. Nilsson M, Valente AJ, Olofsson G, Soderman O, Bonini M. Thermodynamic and kinetic characterization of host-guest association between bolaform surfactants and alpha- and beta-cyclodextrins. J Phys Chem B. 2007;112:11310-6.

16. Sun DZ, Li L, Qiu XM, Liu F, Yin BL. Isothermal titration calorimetry and $1 \mathrm{H}$ NMR studies on host-guest interaction of paeonol and two of its isomers with beta-cyclodextrin. Int $\mathrm{J}$ Pharm. 2006;316:7-13.

17. Blaschke G, Lamparter E, Schluter J. Racemization and hydrolysis of tropic acid alkaloids in the presence of cyclodextrins. Chirality. 1993;5:78-83.

18. Job P. Recherches dur la formation de complexes mineraux en solution, et sur leur stabilite. Ann Chim. 1928;9:113-34.

19. Benesi HA, Hildebrand JH. A spectrophotometric investigation of the interaction of iodine with aromatic hydrocarbons. J Am Chem Soc. 1949;71:2703-7.

20. Asztemborska M, Bielejewska A, Duszczyk K, Sybilska D. Comparative study on camphor enantiomers behavior under the conditions of gas-liquid chromatography and reversed-phase high-performance liquid chromatography systems modified with $\alpha$ - and $\beta$-cyclodextrin. J Chromatogr A. 2000;874:73-80. 